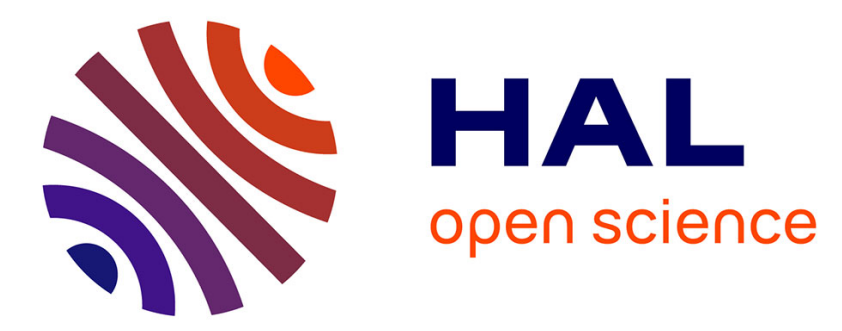

\title{
Optimized terahertz generation via optical rectification in ZnTe crystals
}

Sébastien Vidal, Degert Jérôme, Marc Tondusson, Eric Freysz, Jean Oberle

\section{To cite this version:}

Sébastien Vidal, Degert Jérôme, Marc Tondusson, Eric Freysz, Jean Oberle. Optimized terahertz generation via optical rectification in ZnTe crystals. Journal of the Optical Society of America B, 2014, 31 (1), pp.149. 10.1364/JOSAB.31.000149 . hal-00920309

\section{HAL Id: hal-00920309 \\ https: / hal.science/hal-00920309}

Submitted on 18 Dec 2013

HAL is a multi-disciplinary open access archive for the deposit and dissemination of scientific research documents, whether they are published or not. The documents may come from teaching and research institutions in France or abroad, or from public or private research centers.
L'archive ouverte pluridisciplinaire HAL, est destinée au dépôt et à la diffusion de documents scientifiques de niveau recherche, publiés ou non, émanant des établissements d'enseignement et de recherche français ou étrangers, des laboratoires publics ou privés.

\section{다(1) $\$$}

Distributed under a Creative Commons Attribution - NonCommerciall 4.0 International 


\title{
Optimized terahertz generation via optical rectification in ZnTe crystals
}

\author{
S. Vidal, ${ }^{1,2,3}$ J. Degert, ${ }^{1,2}$ M. Tondusson, ${ }^{1,2}$ E. Freysz, ${ }^{1,2}$ and J. Oberlé $e^{1,2,4}$ \\ ${ }^{1}$ Univ. Bordeaux, LOMA, UMR 5798, F-33400 Talence, France \\ ${ }^{2}$ CNRS, LOMA, UMR 5798, F-33400 Talence, France \\ ${ }^{3}$ e-mail: sebastien.vidal@alphanov.com \\ ${ }^{4}$ e-mail: j.oberle@loma.u-bordeaux1.fr
}

Received October 18, 2013; accepted November 21, 2013;

posted November 27, 2013 (Doc. ID 199750); published December 18, 2013

\begin{abstract}
We report on optimal control of the output energy of terahertz $(\mathrm{THz})$ waves generated by optical rectification of femtosecond pulses in ZnTe crystals. An enhancement by a factor up to 2.4 is obtained with chirped pump pulses. The optimized THz wave also displays a spectral broadening. The influence of the optical pulse shaping on the pump pulse propagation, and consequently on the $\mathrm{THz}$ generation efficiency, is numerically investigated and discussed. (C) 2013 Optical Society of America
\end{abstract}

OCIS codes: (190.7110) Ultrafast nonlinear optics; (320.5540) Pulse shaping.

http://dx.doi.org/10.1364/JOSAB.31.000149

\section{INTRODUCTION}

Optical rectification (OR) of femtosecond laser pulses in zinc blend nonlinear crystals such as ZnTe remains one of the most commonly used techniques for generating ultrashort terahertz (THz) pulses [1]. However, for pump pulses with high peak intensity, saturation effects mainly due to free carriers generated by two-photon absorption (TPA) limit the conversion efficiency. Indeed, TPA not only depletes the pump pulse, but also leads to a reshaping of the $\mathrm{THz}$ waveform that can be attributed to the influence of the photoinduced free carriers on the complex refractive index of the crystal in the THz range [2-5]. Interestingly, it has been shown that, due to free carriers saturation, increasing further the pump pulse fluence leads to a recovering of the quadratic dependence of the $\mathrm{THz}$ power with respect to the pumping fluence usually observed at low intensity [5]. Moreover, dispersion of ultrashort femtosecond Ti: sapphire pulses in thick ZnTe crystal also leads to a decrease of the OR efficiency [2]. Recently, a first step toward the optimization of the $\mathrm{THz}$ wave intensity has been made by adding a negative prechirp to the femtosecond laser pump pulse, leading to an increase of the $\mathrm{THz}$ intensity generated in a ZnTe crystal up to $60 \%$ [6].

Hereafter, we report on the optimal control of the output energy of THz waves generated by OR of amplified femtosecond laser pulses centered at $795 \mathrm{~nm}$ in a $1 \mathrm{~mm}$ thick ZnTe crystal at various pumping power. An increase of the $\mathrm{THz}$ wave energy up to $140 \%$ has been observed with chirped pulses showing that transform-limited (TL) pulses are not well suited when linear and third-order processes are taking place during OR. The optimization procedure lies on an optimal control strategy: the THz energy is used as a feedback signal by a learning evolutionary algorithm, coupled to a pulse shaper, to iteratively improve the shape of the rectified pulses. Moreover, broader $\mathrm{THz}$ spectra are also achieved. Thanks to numerical resolution of the 3D-nonlinear Schrödinger equation, we have investigated the influence of the spectral phase of the optimized pump pulse on its propagation. This numerical resolution makes it possible to account for the evolution of the pump pulse duration, energy, and beam profile during its propagation in the crystal. Our simulations indicate that the evolution of the optimal pump pulse is drastically different at high and low pump pulse fluence.

\section{EXPERIMENTAL SETUP}

The experimental setup, sketched in Fig. 1, is the following: a Ti:sapphire chirped-pulse amplifier (CPA) delivering TL laser pulses of duration $\tau_{p}=35 \mathrm{fs}$ at $795 \mathrm{~nm}$ with a $1 \mathrm{kHz}$ repetition rate is used to generate and detect $\mathrm{THz}$ waves. About $90 \%$ of the output of the CPA is used for the generation of $\mathrm{THz}$ pulses by OR in a $L=1 \mathrm{~mm}$ thick $\langle 110\rangle$ ZnTe crystal. This beam can be spectrally tailored thanks to a pulse shaper [7]. The latter consists in a half-zero-dispersion line made of a 600 grooves $/ \mathrm{mm}$ grating and a $f=600 \mathrm{~mm}$ cylindrical mirror that spatially disperses and focuses all the spectral components of the pulse in the Fourier plane where a programmable single liquid crystal spatial light modulator (LCSLM) with 640 pixels, manufactured by Jenoptik AG, acts

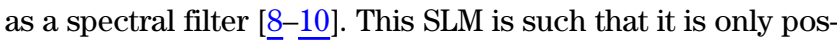
sible to change the spectral phase of the incoming pulse. A plane mirror positioned just after the SLM then folds the line to perform an inverse Fourier transform and reassemble all the spectral components of the shaped pulse. The "shaped" beam then excites the ZnTe emitter at normal incidence. The generated THz wave is imaged into a second $300 \mu \mathrm{m}$ thick $\langle 110\rangle \mathrm{ZnTe}$ crystal by means of four off-axis paraboloidal mirrors. It is then measured via electro-optic (EO) sampling in this crystal using a weak probe TL pulse [11], the EO signal being detected by ellipsometry. For the purpose of the optimization of the $\mathrm{THz}$ generation, we also used a Golay Cell (GC-1P, TYDEX) to measure the THz energy generated as a 


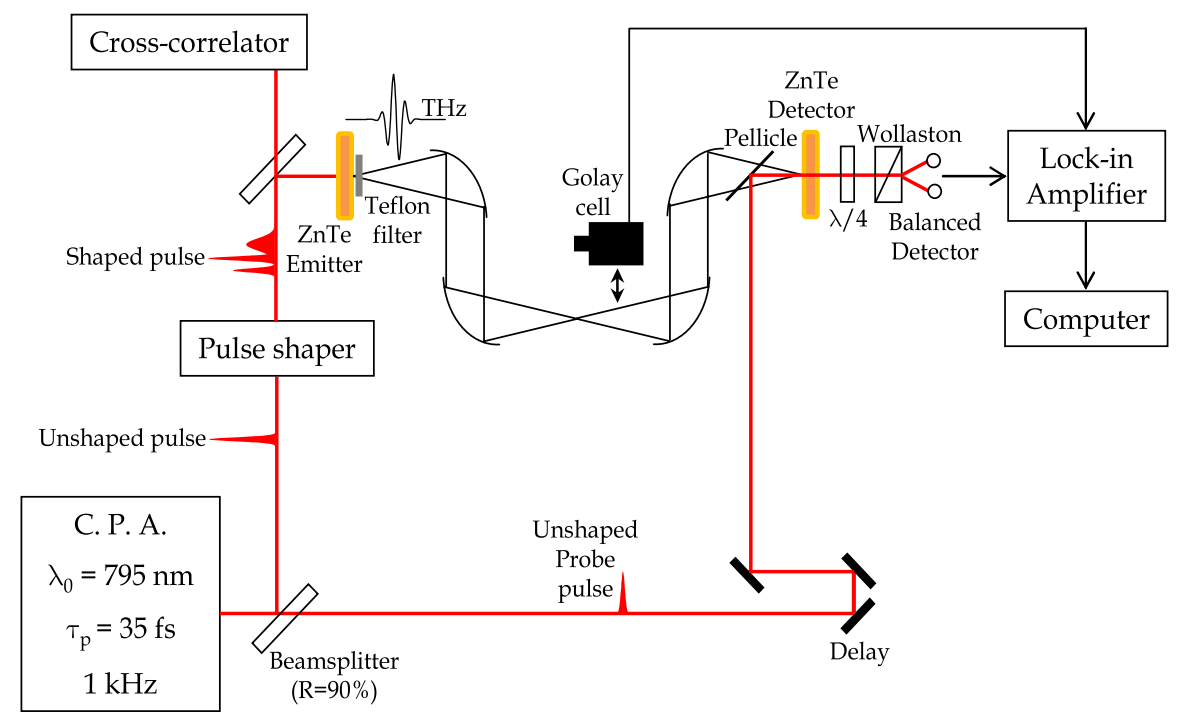

Fig. 1. Experimental setup developed for the optimization of the THz generation in a $1 \mathrm{~mm}$ thick ZnTe crystal.

function of the "shape" of the optical pulse. It was inserted at the focal point lying between the emitter and the detector.

\section{OPTIMAL CONTROL}

To perform the optimization of the THz generation by OR, we chose an approach based on a feedback-controlled shaping of the optical pump pulse. Such a strategy was proposed by Judson and Rabitz two decades ago [12], and was implemented experimentally few years later [13-15]. Since then, it has been proven successful in controlling different types of processes [16].

Here, we excite a ZnTe crystal with a shaped ultrashort laser pulse, and we monitor the energy of the THz pulse generated by OR. The latter signal serves as a feedback for an optimization algorithm that drives the optical pulse shaper. The optimization process is performed thanks to an evolutionary algorithm whose principle is explained in detail in [17]. For each loop of the optimization procedure, we use a population consisting of 16 pulses, each pulse being characterized by four parameters, controlled by the pulse shaper, corresponding to the coefficients of the expansion of the spectral phase in a polynomial of degree five, that is

$$
\varphi(\omega)=\sum_{n=2}^{5} \frac{1}{n !} \varphi^{(n)}\left(\omega-\omega_{0}\right)^{n},
$$

where $\omega_{0}$ is the carrier angular frequency of the pump pulse and the derivatives, in this expansion, are respectively the group-delay dispersion GDD $\left(\varphi^{(2)}\right)$, the third-order dispersion TOD $\left(\varphi^{(3)}\right)$, and so on. Such a parameterization of the optical pulses was adopted in order to reduce the size of the parameter space to explore, thus simplifying the task of the algorithm [18]. Note that, in the same spirit, we also impose boundary conditions on the four phase parameters values. Prior to any experiment in the $\mathrm{THz}$ range, we tested our setup by optimizing the second-harmonic generation of intentionally chirped optical pulses in a $100 \mu \mathrm{m}$ thick beta barium borate (BBO) crystal cut for type I phase matching at $795 \mathrm{~nm}$. The values obtained for the spectral phase parameters were in full agreement with those deduced from the characteristics of the dispersive medium introduced on the path of the pulse. For the optimization of the OR process in ZnTe, we chose to maximize the ratio, denoted hereafter as $R_{\text {opt }}$, between the THz energy generated by a shaped pulse with respect to the THz energy generated by a TL pulse. So, for each optimization run, the evolutionary algorithm starts with all the parameters of the optical pulse set to zero (i.e., with a TL pulse), then generates randomly 16 pulses, and initiates the optimization process. The algorithm was found to converge the most rapidly toward the most suited pulse for OR when the following criteria were applied to generate a population for each loop of the optimization process: as a basis for the next generation population, we kept only the four best pulses of the previous loop, and generated the 12 remaining pulses by means of mutation and cloning of the survivors. Recombination of two survivors was ineffective in our case.

\section{EXPERIMENTAL RESULTS}

Our results show that optimized laser pulses are mainly dominated by the GDD term in the phase expansion and are not affected by the relative low values found for the higher spectral phase orders. For a given pump pulse energy, the best optical to $\mathrm{THz}$ energy conversion efficiency is finally achieved with an appropriately chirped pulse. More precisely, the optimized chirped pulses have duration ranging from 90 to $160 \mathrm{fs}$. They are obtained by adding negative GDD, in between $-1050 \mathrm{fs}^{2}$ and $-1750 \mathrm{fs}^{2}$, for input pulses with fluences varying from 0.018 to $2.1 \mathrm{~mJ} / \mathrm{cm}^{2}$. Figure 2 displays the evolution of the ratio $R_{\text {opt }}$ at each step during the iteration process for two different input fluences. An enhancement of $R_{\text {opt }}$ up to 2.4 is achieved after 7 to 15 generations, depending on the incident laser pulse fluence. The reproducibility of the convergence curve in different runs was pretty good, the final values of the coefficients varying slightly from one run to the other.

Table 1 summarizes the THz energy enhancement factor obtained for different input fluences and the corresponding optimized GDD values. An increase of $R_{\text {opt }}$ with increasing pump fluences is observed, up to a maximum reached for about $1.75 \mathrm{~mJ} / \mathrm{cm}^{2}$. The corresponding optimized $\mathrm{THz}$ pulse energy achieved for this pump fluence was $23 \mathrm{~nJ}$. 


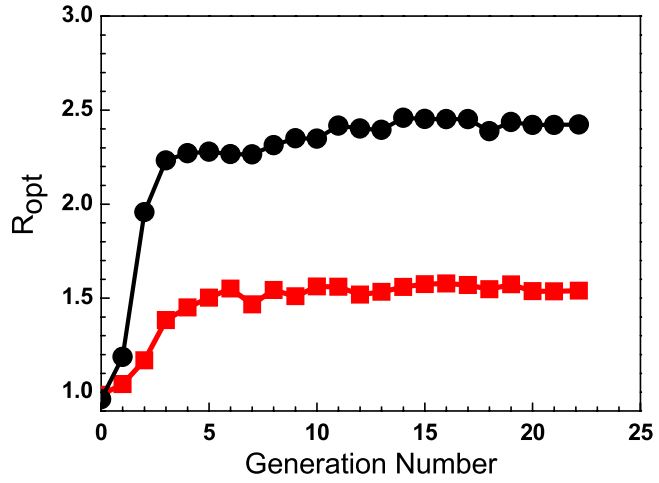

Fig. 2. Typical convergence curves for the optimization of the THzwave energy generated in a $1 \mathrm{~mm}$ thick ZnTe crystal excited by pulses with fluences of $0.03 \mathrm{~mJ} / \mathrm{cm}^{2}$ (squares) and $1.75 \mathrm{~mJ} / \mathrm{cm}^{2}$ (dots), respectively.

Moreover, as shown by Fig. 3 , the THz power spectra given by optimized chirped pump pulses are broaden with respect to the ones given by TL pulses. Note also that the THz spectrum after optimization is similar to the one generated at very low pump fluences [2].

\section{NUMERICAL ANALYSIS}

To have a better understanding on the evolution of the pump pulse during its propagation through the $1 \mathrm{~mm}$ thick ZnTe crystal, we have numerically solved its wave equation, namely the following 3D-nonlinear Schrödinger equation [19]:

$$
\begin{aligned}
& \frac{\partial E(x, y, z, t)}{\partial z}-\frac{i}{2 k_{0}} \Delta_{t} E(x, y, z, t)+\frac{i}{2} k_{0}^{\prime \prime} \frac{\partial^{2} E(x, y, z, t)}{\partial t^{2}} \\
& =-\frac{1}{2} \beta|E(x, y, z, t)|^{2} E(x, y, z, t) .
\end{aligned}
$$

Here, the optical pulse propagates along the $z$ axis, its electric field $E(x, y, z, t)$ being given by

$$
E(x, y, z, t)=u(x, y, z) \varepsilon(z, t) \exp \left[i\left(\omega_{0} t-k_{0} z\right)\right],
$$

where $u(x, y, z)$ and $\varepsilon(z, t)$ are respectively the spatial and temporal amplitudes of the laser pulse, $\omega_{0}$ its carrier frequency (corresponding to a central wavelength $\lambda_{0}=2 \pi c / \omega_{0}, c$ is the velocity of light in vacuum), and $k_{0}$ the mean wave vector $\left(k_{0}=\omega_{0} n_{0} / c\right.$, where $n_{0}$ is the refractive index of ZnTe at $\lambda_{0}=795 \mathrm{~nm}$ ). In Eq. (2), $k_{0}^{\prime \prime}=2.2 \mu \mathrm{m}^{-1} \cdot \mathrm{fs}^{2}$ is the dispersion parameter at $\lambda_{0}[\underline{6}], \Delta_{t}=\partial^{2} / \partial x^{2}+\partial^{2} / \partial y^{2}$, and $\beta$ is the TPA coefficient. Note that we have not considered in Eq. (2) the influence of self-phase modulation since we did not observed it in our experiment even for high input intensities. Due to the weak optical to $\mathrm{THz}$ conversion efficiency

Table 1. THz Energy Enhancement Factor $\boldsymbol{R}_{\mathrm{opt}}$ for Different Input Fluences $J$ and the Corresponding Optimized Values of the GDD

\begin{tabular}{lcc}
\hline$J\left(\mathrm{~mJ} / \mathrm{cm}^{2}\right)$ & $\varphi^{(2)}\left(\mathrm{fs}^{2}\right)$ & $R_{\text {opt }}$ \\
\hline 0.018 & $-1050 \pm 100$ & $1.3 \pm 0.1$ \\
0.035 & $-1100 \pm 100$ & $1.60 \pm 0.15$ \\
0.35 & $-1700 \pm 100$ & $2.10 \pm 0.15$ \\
1.75 & $-1750 \pm 150$ & $2.40 \pm 0.15$ \\
2.1 & $-1800 \pm 150$ & $2.40 \pm 0.15$ \\
\hline
\end{tabular}

(about $10^{-5}$ ), we have also neglected the coupling between the pump and the THz pulses. Moreover, Eq. (2) does not account for the refractive index change induced in the optical domain by the free carriers due to its negligible value [2]. Considering spatially and temporally Gaussian shaped pulses, we have solved Eq. (2) using a split-step Fourier transform method [20]. Note that TPA is usually described using the following phenomenological law:

$$
\frac{\partial I(x, y, z, t)}{\partial z}=-\beta^{I} I^{2}(x, y, z, t)
$$

with $\beta^{I}=2 \beta / \varepsilon_{0} c n_{0}$ ( $\varepsilon_{0}$ is the vacuum permittivity) and $I(x, y, z, t)$ the input intensity. To account for the saturation of TPA, we have fitted measurements of the transmitted intensity at $795 \mathrm{~nm}$ versus input intensity using the following intensity-dependent absorption coefficient model [21]:

$$
\beta^{I}=\frac{\beta_{0}}{1+I(x, y, z, t) / I_{\mathrm{sat}}}
$$

where $\beta_{0}$ and $I_{\text {sat }}$ are, respectively, the TPA coefficient at very low intensity and the saturation intensity. We obtained $\beta_{0}=$ $4 \mathrm{~cm} / \mathrm{GW}$ and $I_{\text {sat }}=7 \mathrm{GW} / \mathrm{cm}^{2}$ in agreement with [21]. As indicated by our experimental results, it is mainly the introduction of GDD that contributes to the optimization of $\mathrm{THz}$ generation. In the spectral domain, GDD of the input pulse writes as follows:

$$
\varepsilon(0, t)=\mathrm{FT}^{-1}\left\{E_{\mathrm{TL}}(0, \omega) \exp \left[i \frac{\varphi^{(2)}}{2} \omega^{2}\right]\right\},
$$

with $E_{\mathrm{TL}}(0, \omega)$ the spectral amplitude of the input TL pulse.

\section{DISCUSSION}

Thanks to the numerical analysis, we have determined the evolution of the pump pulse intensity, energy, and duration during the propagation of the TL and the optimized pulses through the $1 \mathrm{~mm}$ thick ZnTe crystal (cf. Figs. $\underline{4}$ and $\underline{5}$ ).

At low input pulse fluence, the depletion of the TL pulse due to TPA is negligible [cf. Fig. 4(c)] and thus the reduction of its

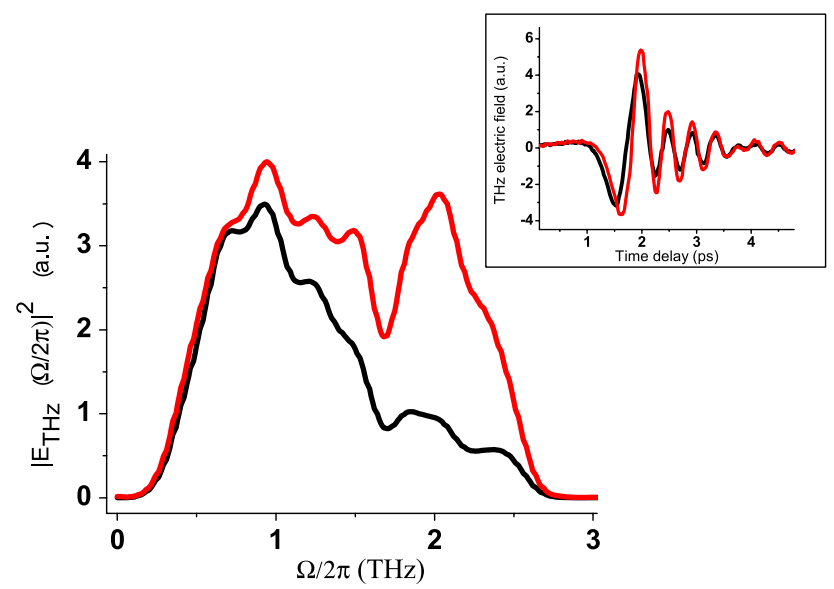

Fig. 3. THz power spectra and corresponding temporal waveforms (inset) generated in a $1 \mathrm{~mm}$ thick ZnTe crystal excited by a TL pulse $\left(\varphi^{(2)}=0 \mathrm{fs}^{2}\right)$ (dark lines) and an optimized one $\left(\varphi^{(2)}=-1700 \mathrm{fs}^{2}\right)$ (red lines), both having an input fluence of $0.35 \mathrm{~mJ} / \mathrm{cm}^{2}$. 

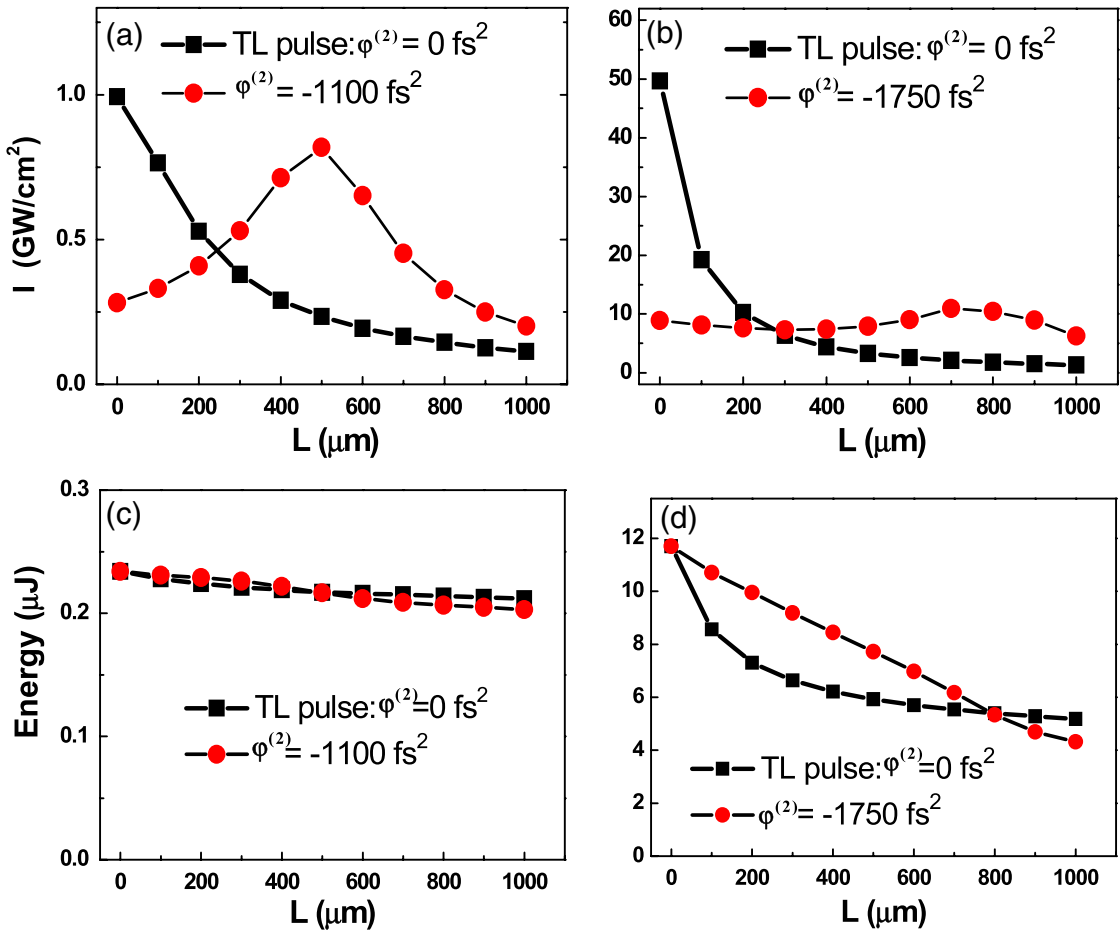

Fig. 4. Evolution of the laser pulse intensity during its propagation through a $1 \mathrm{~mm}$ thick crystal (a) for an initial $0.035 \mathrm{~mJ} / \mathrm{cm}^{2} \mathrm{TL}$ pulse $\left(\varphi^{(2)}=0 \mathrm{fs}^{2}\right)$ and for the optimized one (GDD $\left.\varphi^{(2)}=-1100 \mathrm{fs}^{2}\right)$; (b) for an initial $1.75 \mathrm{~mJ} / \mathrm{cm}^{2}$ TL pulse, and the corresponding optimized pulse $\left(\varphi^{(2)}=-1750 \mathrm{fs}^{2}\right)$. (c) Evolution of the laser pulse energy for an initial $0.035 \mathrm{~mJ} / \mathrm{cm}^{2} \mathrm{TL}$ pulse and for the optimized one. (d) Evolution of the laser pulse energy for an initial $1.74 \mathrm{~mJ} / \mathrm{cm}^{2} \mathrm{TL}$ pulse and for the optimized one.

peak intensity observed in Fig. $\underline{4(a)}$ is mainly due to the increase of the pulse duration induced by the dispersion of the crystal (Fig. 5). In agreement with [6], the optimized value of the GDD leads to maximize the pulse intensity close to the center of the crystal [cf. Fig. 4(a)]. Hence the pump pulse experiences a recompression during its propagation toward the center of the crystal (cf. Fig. 5). Note that at the exit of the crystal, the depletion of the TL and the optimized pulses is almost similar [cf. Fig. 4(c)]. This indicates that the optimized pulse just compensates for the dispersion of the ZnTe, leading to an increase of the overall peak intensity [cf. Fig. 4(a)].

Things are quite different under high input pump pulse fluence. In that case, our results clearly show that TPA strongly

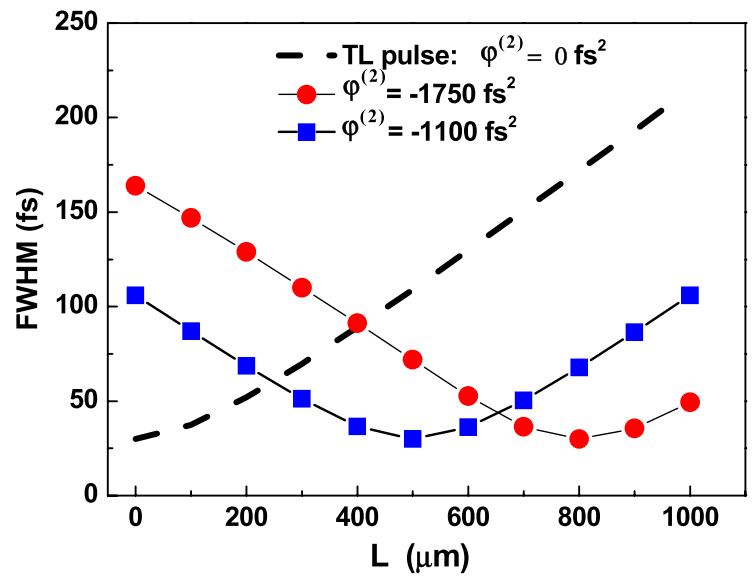

Fig. 5. Evolution of the Gaussian pulse duration (FWHM in intensity) during the propagation for a $35 \mathrm{fs}, 0.035 \mathrm{~mJ} / \mathrm{cm}^{2}$ TL pulse (dashed line) and for pulses with GDD $\varphi^{(2)}=-1100 \mathrm{fs}^{2}$ (blue squares) and $\varphi^{(2)}=-1750 \mathrm{fs}^{2}$ (red circles). depletes the pump pulse [cf. Fig. $\underline{4(\mathrm{~d})}$ and Table 2] : about $60 \%$ of the pulse energy is absorbed by the crystal! As indicated in Table 2 , the saturation of TPA occurs about $0.35 \mathrm{~mJ} / \mathrm{cm}^{2}$, in accordance with the value for which $R_{\text {opt }}$ reaches a maximum (Table 1). Note the good agreement between experimental and numerical results. Besides absorption of the input pulse by TPA, the photoinduced free carriers strongly absorb the THz energy and therefore impact both the output THz energy and spectrum [2]. Compared to the optimized input pulse injected in the crystal at low fluence, the optimum pulse shape is found for larger negative GDD values. Hence, the optimal pulse duration occurs toward the rear face of the crystal (cf. Fig. 5). Note that the depletion rate of this shaped pulse can be considered as constant along the crystal [cf. Fig. 4(d)] and that the total absorption is, surprisingly, greater than the one obtained with the TL pulse [cf. Fig. 4(d) and Table 2]. Further, the peak intensity of the resulting pulse is significantly lower than the one of the TL pulse at the input of the crystal, thus minimizing the creation of photocarriers. It has a

Table 2. Laser Pump Energy Fraction Absorbed by TPA in the ZnTe Crystal

Pump Energy Fraction Absorbed by TPA TL Pulse/Optimized Pulse

\begin{tabular}{lcc}
\cline { 2 - 3 } $\begin{array}{l}\text { Incident Pump } \\
\text { Pulse Fluence } J\end{array}$ & Experimental & Simulation \\
\hline $0.035 \mathrm{~mJ} / \mathrm{cm}^{2}$ & $7 \% / 10 \%$ & $9 \% / 13 \%$ \\
$0.18 \mathrm{~mJ} / \mathrm{cm}^{2}$ & $35 \% / 47 \%$ & $34 \% / 41 \%$ \\
$0.35 \mathrm{~mJ} / \mathrm{cm}^{2}$ & $40 \% / 46 \%$ & $39 \% / 45 \%$ \\
$1.75 \mathrm{~mJ} / \mathrm{cm}^{2}$ & $58 \% / 65 \%$ & $56 \% / 63 \%$ \\
$2.1 \mathrm{~mJ} / \mathrm{cm}^{2}$ & $61 \% / 67 \%$ & $59 \% / 65 \%$ \\
\hline
\end{tabular}




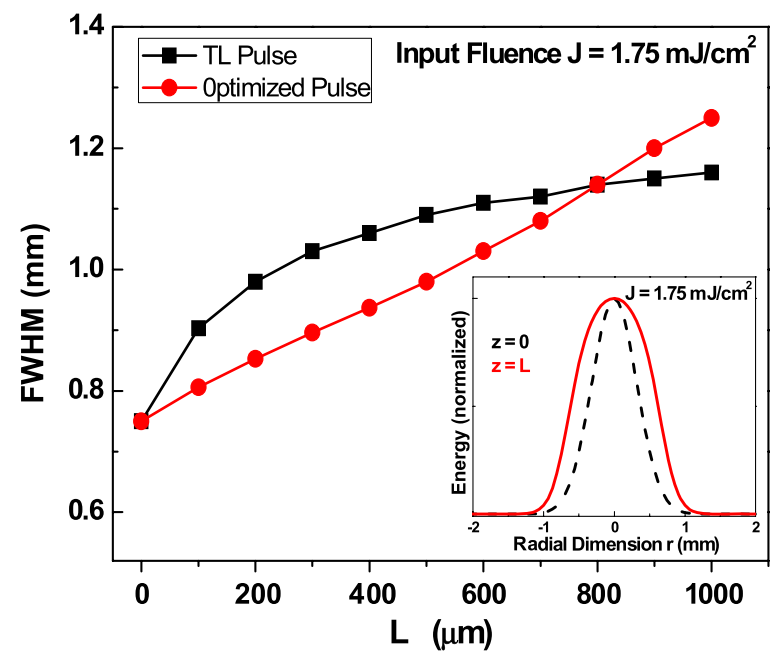

Fig. 6. Evolution of the diameter (FWHM in intensity) of the laser beam along the crystal for the TL and the optimized pulse under high input fluence. Inset: beam profile at the input and exit of the crystal for a TL pulse with high fluence $\left(r=\sqrt{x^{2}+y^{2}}\right)$.

constant value along the crystal [cf. Fig. 4(b)], keeping almost steady the amplitude of THz nonlinear polarization. The combination of all these effects leads to an enhancement of the $\mathrm{THz}$ energy around $140 \%$ and a $\mathrm{THz}$ spectral broadening. Consequently, we have found a compromise between the requirement of intense pump pulses for efficient $O R$, the drawbacks inherent to TPA (depletion of the pump and THz absorption due to the photoinduced free carriers), and the compensation of the dispersion.

We have also investigated the evolution of the spatial mode of the pump pulse during its propagation along the ZnTe crystal. As the Rayleigh's length is much greater than the length of the crystal, all effects of diffraction are negligible. Figure 6 displays the evolution of the diameter (FWHM in intensity) of the laser beam along the crystal as well as the beam profile at the input and exit of the crystal at high input fluence. We noticed that, at low laser input fluence, the laser beam is almost not affected by TPA and keeps a Gaussian profile during the propagation. However, under high pump pulse fluence, TPA occurs preferentially at the center of the beam leading to a slight deformation of the beam profile (cf. inset of Fig. 6). Figure 6 also indicates that the beam diameter of the TL pulse increases more rapidly than the diameter of the optimized one, the latter increasing almost linearly along the crystal. This behavior closely follows the evolution of the pump energy [cf. Fig. 4(d)], and, combined with the evolution of the pulse duration (Fig. 5), explains the behavior of the peak intensity within the crystal.

\section{CONCLUSION}

We have shown that, thanks to an optimal control of the output energy of THz waves generated by OR in ZnTe crystals, we were able to enhance this quantity by a factor 2.4. For high pump pulse fluence, such an optimal control strategy also results in a broadening of the generated THz spectrum. Our data show that the spectral phase of the optimal pump pulse is mainly dominated by group delay dispersion. To account for this ensemble of results, the propagation of the pump pulse during its propagation in the ZnTe crystal has been numerically solved using the 3D-nonlinear Schrödinger equation. It indicates that the optimal pulse shape results from a compromise between the requirement of pump pulses intense enough to generate efficiently $\mathrm{THz}$ by $\mathrm{OR}$, a reduction of both the influence of pump depletion by TPA and the impact of the photoinduced free carriers on $\mathrm{THz}$, and, finally, the compensation of the pump pulse broadening due to dispersion.

\section{REFERENCES}

1. K. Reimann, "Table-top sources of ultrashort THz pulses," Rep. Prog. Phys. 70, 1597-1632 (2007).

2. S. Vidal, J. Degert, M. Tondusson, J. Oberle, and E. Freysz, "Impact of dispersion, free carriers and two-photon absorption on the generation of intense THz pulses in ZnTe crystals," Appl. Phys. Lett. 98, 191103 (2011).

3. S. M. Harrel, R. L. Milot, J. M. Schleicher, and C. A. Schmuttenmaer, "Influence of free-carrier absorption on terahertz generation from ZnTe(110),” J. Appl. Phys. 107, 033526 (2010).

4. Z. Y. Zhao, S. Hameau, and J. Tignon, "THz generation by optical rectification and competition with other nonlinear processes," Chin. Phys. Lett. 25, 1868-1870 (2008).

5. S. A. Ku, C. M. Tu, W.-C. Chu, C. W. Luo, K. H. Wu, A. Yabushita, C. C. Chi, and T. Kobayashi, "Saturation of the free carrier absorption in ZnTe crystals," Opt. Express 21, 13930-13937 (2013).

6. D. N. Erschens, D. Turchinovich, and P. U. Jepsen, "Optimized optical rectification and electro-optic sampling in ZnTe crystals with chirped femtosecond laser pulse," J. Infrared Millim. Terahertz Waves 32, 1371-1381 (2011)

7. S. Vidal, J. Degert, J. Oberlé, and E. Freysz, "Femtosecond optical pulse shaping for tunable terahertz pulses generation," J. Opt. Soc. Am. B 27, 1044-1050 (2010).

8. G. Stobrawa, M. Hacker, T. Feurer, D. Zeidler, M. Motzkus, and F. Reichel, "A new high resolution femtosecond pulse shaper," Appl. Phys. B 72, 627-630 (2001).

9. A. M. Weiner, "Femtosecond pulse shaping using spatial light odulators," Rev. Sci. Instrum. 71, 1929-1960 (2000).

10. A. Monmayrant and B. Chatel, "New phase and amplitude high resolution pulse shaper," Rev. Sci. Instrum. 75, 2668-2671 (2004).

11. A. Nahata, A. S. Weling, and T. F. Heinz, "A wideband coherent terahertz spectroscopy system using optical rectification and electro-optic sampling," Appl. Phys. Lett. 69, 2321-2323 (1996).

12. R. S. Judson and H. Rabitz, "Teaching laser to control molecules," Phys. Rev. Lett. 68, 1500-1503 (1992).

13. D. Meshulach, D. Yelin, and Y. Silberberg, "Adaptive ultrashort pulse compression and shaping," Opt. Commun. 138, 345-348 (1997).

14. T. Baumert, T. Brixner, V. Seyfried, M. Strehle, and G. Gerber, "Femtosecond pulse shaping by an evolutionary algorithm with feedback," Appl. Phys. B 65, 779-782 (1997).

15. C. J. Bardeen, V. V. Yakovlev, K. R. Wilson, S. D. Carpenter, P. M. Weber, and W. S. Warren, "Feedback quantum control of molecular electronic population transfer," Chem. Phys. Lett. 280, 151-158 (1997).

16. D. Goswami, "Optical pulse shaping approaches to coherent control," Phys. Rep. 374, 385-481 (2003).

17. D. Zeidler, S. Frey, K.-L. Kompa, and M. Motzkus, "Evolutionary algorithms and their application to optimal control studies," Phys. Rev. A 64, 023420 (2001).

18. J. Kunde, B. Baumann, S. Arlt, F. Morier-Genoud, U. Siegner, and U. Keller, "Optimization of adaptive feedback control for ultrafast semiconductor spectroscopy,” J. Opt. Soc. Am. B 18, 872-881 (2001).

19. L. Yin and G. Agrawal, "Impact of two-photon absorption on self-phase modulation in silicon waveguides," Opt. Lett. 32, 2031-2033 (2007).

20. J. A. C. Weideman and B. M. Herbst, "Split-step method for the solution of the nonlinear Schrodinger equation," SIAM J. Numer. Anal. 23, 485-507 (1986).

21. B. Gu, Y.-X. Fan, J. Chen, H.-T. Wang, J. He, and W. Ji, "Z-scan theory of two-photon absorption saturation and experimental evidence," J. Appl. Phys. 102, 083101 (2007). 